\section{$A B C$ der Strümpfe}

- Immer wieder werden sie miteinander verwechselt, dabei unterscheiden sie sich ganz erheblich in ihrer Wirkung und ihren Einsatzgebieten: Medizinische Kompressionsstrümpfe, Thromboseprophylaxestrümpfe und Stützstrümpfe. Eine Hilfestellung gibt jetzt die Herstellervereinigung für Kompressionstherapie und orthopädische Hilfsmittel:

Medizinische Kompressionsstrümpfe gelten als Basisbehandlung aller Erkrankungen des Venen- und Lymphgefäßsystems. Sie üben einen definierten Druck auf das Gewebe und die Gefäße aus. Erweiterte Venen werden auf ihren normalen Durchmesser zusammengepresst, noch nicht zerstörte Venenklappen schließen wieder und werden ihrer Funktion als Rückstauventil gerecht. Das Blut kann ungehindert zum Herzen zurückfließen und staut sich nicht mehr in den Beinen. Medizinische Kompressionsstrümpfe werden in vier verschiedene Kompressionsklassen eingeteilt, die sich in der Intensität des Andrucks in Ruhe unterscheiden. Zur Thromboseprophylaxe bei bettlägerigen Patienten sind medizinische Kompressionsstrümpfe nicht geeignet. Sie entfalten ihre volle Wirkung am besten, wenn der Patient sich bewegt. Thromboseprophylaxestrümpfe (auch Antithrombosestrümpfe) werden bei bettlägerigen und frisch operierten $\mathrm{Pa}$ tienten zur Prophylaxe von Venenthrombosen eingesetzt. Für aufrecht gehende und sitzende Patienten sind sie wegen des niedrigen Drucks und der Strickweise nicht geeignet. Antithrombosestrümpfe werden aus Erkennungsgründen nur in Weiß gestrickt.

Stützstrümpfe dürfen nicht mit Kompressionsstrümpfen verwechselt werden. Sie eignen sich ausschließlich für Venengesunde zur Vorbeugung gegen schwere, müde Beine bei langem Stehen oder Sitzen und gegen Reisethrombose. Als Prophylaxemaßnahme werden diese Produkte nicht von den Krankenkassen bezahlt und können bei einer bestehenden Venenerkrankung einen Kompressionsstrumpf auch nicht ersetzen.

www.eurocom-info.de

Drei-Länder-Wundpreis 2015

\title{
Die Bewerbungsfrist läuft
}

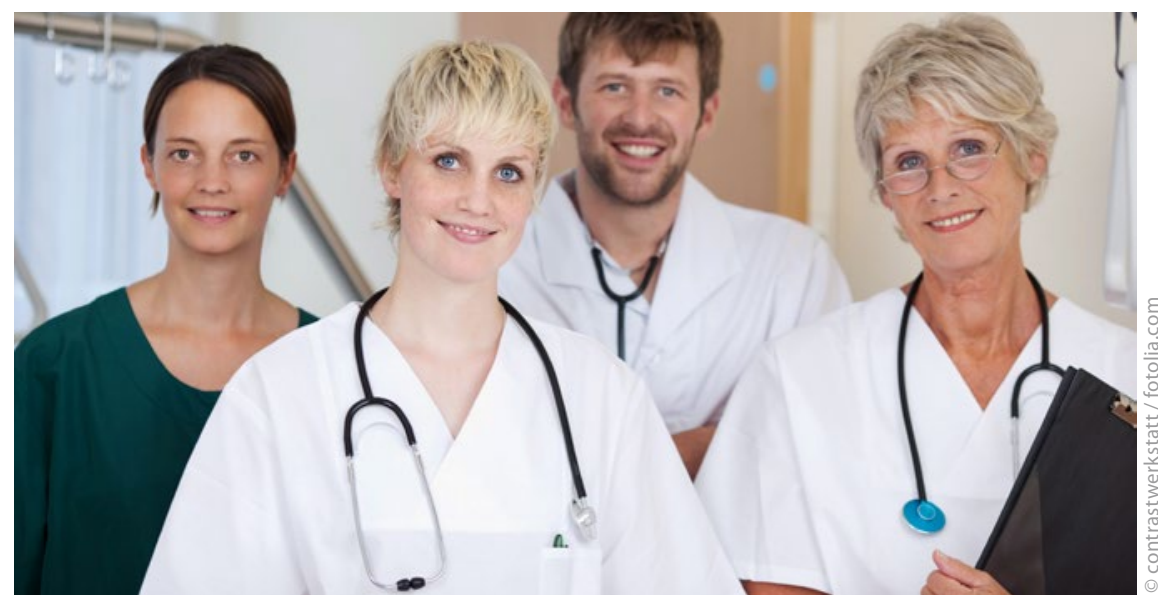

- Die gemeinnützige Fondation URGO lobt zum zweiten Mal den Drei-LänderWundpreis für Deutschland, Österreich und die Schweiz aus. Die Stiftung vergibt das Preisgeld bevorzugt für Projekte mit einem fachübergreifenden medizinisch-pflegerischen Ansatz. Auch der länderübergreifende Erfahrungsaustausch soll gefördert und unterstützt werden. Für den Drei-LänderWundpreis 2015 sind verschiedene Themenschwerpunkte festgelegt worden: Von der Grundlagen- und Anwendungsforschung auf dem Gebiet der Wundheilung, über Schulungs- und Fortbildungskonzepte in der Wundbehandlung bis hin zu Projekten zur Rezidivprophylaxe bei chronischen Wunden. Teilnahmeberechtigt sind examinierte Pflegefachkräfte, Pflegewissenschaftler, Ärzte aller Fachgebiete sowie Doktoranden der Medizin. Bewerber können ihre Unterlagen bis zum 31.12.2015 einreichen. Die Arbeiten werden von einer unabhängigen Expertenjury aus Medizin und Pflege bewertet. Der Drei-LänderWundpreis 2015 ist mit insgesamt $10.000 €$ dotiert. Ausführliche Informationen unter:

www.urgo.de

\section{Expertengruppe gegründet \\ Kompressionstherapie im Fokus}

— Mit dem medizinischen Fortschritt gehen bisweilen anerkannte Behandlungsmethoden aus dem öffentlichen Bewusstsein verloren. Dazu gehört auch die Kompressionstherapie. Eine 10-köpfige interdisziplinäre Expertengruppe will dem entgegenwirken. Ihr Ziel: Das Bewusstsein für die Wirksamkeit und den Nutzen der Kompressionstherapie zu stärken. Koordiniert wird die Gruppe vom Starnberger Medical Data Institute, das hierzu eigens das Ressort Kompressionstherapie gegründet hat.

Prof. Joachim Dissemond, einer der Ressortleiter, bedauert, dass die Kenntnisse über die Vielfalt der Kompressionstherapie und andere entstauende Verfahren sowie deren breiten klinisch Nutzen schrittweise verlo- ren gingen. Aus Unkenntnis würden sie nicht angewandt und es fehlten die passenden Fortbildungsangebote für Ärzte und Pflegekräfte. Dabei habe die Kompressionstherapie in der Medizin eine lange Tradition und im Bereich der Erkrankungen von Venen- und Lymphgefäßen einen festen Stellenwert. Andererseits sieht die Medizin sich mit einer Vielzahl von Herausforderungen konfrontiert, für die die Kompressionstherapie von großem Nutzen ist. Dazu gehören neben den klassischen Indikationen auch die Therapie der chronischen Rechtsherzinsuffizienz, die Adipositas und die Inaktivitätsödeme bei älteren Pflegebedürftigen.

www.md-institute.com 